\title{
L-BAND DIGITAL AERONAUTICAL COMMUNICATIONS SYSTEM (LDACS) ACTIVITIES IN SESAR2020
}

\author{
C. Rihacek, B. Haindl, Frequentis AG, Vienna, Austria \\ P. Fantappie, S. Pierattelli, Leonardo S.p.a., Italy \\ T. Gräupl, M. Schnell, German Aerospace Center, Oberpfaffenhofen, Germany \\ N. Fistas, EUROCONTROL, Brussels, Belgium
}

\begin{abstract}
Air traffic management communication shall transition from analog VHF voice communication to more spectrum efficient digital data communication. In Europe this transition shall be realized, among others, by the development and implementation of the L-band Digital Aeronautical Communications System (LDACS). The objective of this paper is to provide a status report on the current progress of LDACS research and development within SESAR2020. In particular, we present the objectives of SESAR2020 project "PJ.14-02-01 FCI Terrestrial Data Link", the proposed LDACS high-level architecture, the planned technical validation exercises, and the status of the ongoing LDACS standardization activities: The objective of the SESAR2020 project is to develop fully functional LDACS prototypes and to use them for validation of the air interface requirements and the support of the ATN IPS infrastructure. The LDACS architecture has been defined as a complete set of network functions needed to provide radio access to an LDACS subscriber i.e. an aircraft. LDACS standardization is currently under way in ICAO and is planned to start in EUROCAE later this year.
\end{abstract}

\section{Introduction}

One of the main pillars of the modern air traffic management (ATM) system is the existence of a communication infrastructure that enables efficient aircraft guidance and safe separation in all phases of flight. Although current systems are technically mature, they are suffering from the VHF band's increasing saturation in high density areas. Therefore, aviation globally and in the European Union (EU) in particular, strives for a sustainable modernization of the aeronautical communication infrastructure.

In the long-term, ATM communication shall transition from analog VHF voice and VDL2 communication to more spectrum efficient digital data communication. The European ATM Master Plan [1] foresees this transition to be realized, among others, by the development and implementation of the L-band Digital Aeronautical Communications System (LDACS). It is important to highlight that LDACS is one of the three new and key components of the Future Communications Infrastructure (FCI), which foresees the coordinated use of legacy and new technologies (LDACS, AeroMACS and New SATCOM) to meet the future communication requirements and support B2 and B3 ATS services.

The development of LDACS has already made substantial progress in the Single European Sky ATM Research (SESAR) framework, and is currently being continued in the follow-up program, SESAR2020. A key objective of the SESAR activities is to develop, implement and validate a modern aeronautical data link able to evolve with aviation needs over the longterm. To this end, an LDACS specification has been produced [2] and is currently updated; transmitter demonstrators were developed to test the spectrum compatibility of LDACS with legacy systems operating in the L-band [3]; and the overall system performance was analyzed by computer simulations, indicating that LDACS can fulfil the identified requirements. In addition, LDACS standardization within the ICAO framework started in December 2016. The ICAO standardization group is working closely together with SESAR and is aiming at having initial Standards and Recommended Practices (SARPs) ready by end of this year (2018).

The objective of this paper is to provide a status report on the current progress of LDACS research and development within SESAR2020. In particular, we present the objectives of SESAR2020 project "PJ.14-02-01 FCI Terrestrial Data Link", the proposed LDACS high-level architecture, the planned technical validation exercises, and the status of the LDACS standardization activities. 


\section{Background}

Communications, Navigation and Surveillance (CNS) systems provide the invisible and often unappreciated infrastructure which is essential for air traffic management. CNS enables efficient navigation and safe separation in all phases of flight. The SESAR concept of operations aims to dramatically increase the exchange of information between airspace users, air traffic control and flow management. This is expected to improve the quality of service to the travelling public, whilst reducing cost and environmental impact. Although current CNS systems are mature and generally providing a good service, they have not yet fully transitioned from analog to digital technologies. Such a shift is needed to meet the challenges of the coming years; to accommodate higher levels of traffic and improve operational efficiency.

\section{SESAR2020}

Building on the experience gained in the first phase of SESAR (SESAR1), SESAR2020 will develop an integrated suite of CNS solutions to meet the operational requirements of the ATM system in the short, medium and long term, with technologies that are consistent with the European ATM Master Plan and the ICAO Global Air Navigation Plan [4].

SESAR2020 developments are based on four Key Features, as described in the European ATM Master Plan:

- Optimized ATM Network services

- Advanced air traffic services

- High-Performing airport operations

- Enabling aviation infrastructure

Project 14 "PJ14 Essential and Efficient Communication Navigation and Surveillance Integrated System" (EECNS), as one of the enabling projects within SESAR2020, aims at providing advanced, integrated and rationalized aviation infrastructure for CNS, supporting the first three Key Features, and providing the underlying technical capabilities to meet the operational improvements.

\section{SESAR2020 Project PJ.14-02-01 "FCI Terrestrial Data Link"}

The more stringent ATM performance requirements, due to the change of ATM to a $4 \mathrm{D}$ trajectory based concept, raises the need to introduce an appropriate data link solution to support the air traffic services evolution according to the ICAO technology roadmaps and the ATM key improvement areas of SESAR. In particular, the 4D trajectory management operational concept needs to be supported by a reliable, scalable, modular and efficient data link technology.

Within SESAR PJ14 the (sub-)project ${ }^{1}$ PJ.1402-01 "FCI Future Terrestrial Data Link" has the objective to develop and standardize the candidate future terrestrial data link system LDACS. The goal of PJ.14-02-01 is to progress with the development and validation of the LDACS technology. This includes security and digital voice concepts, and will contribute to the development of a harmonized global standard. To achieve these, the project will develop and verify a fully functional LDACS prototype and assess the impact on other systems. The prototype will support ATS B2 applications using the ATN/IPS protocols.

\section{Objectives of SESAR2020 Project PJ.14-02-01 "FCI Terrestrial Data Link"}

The definition of the future terrestrial data link LDACS $^{2}$ is based on the work already performed on LDACS in SESAR1 ${ }^{3}$ and is aligned with international standardisation activities. The activities planned in PJ.14-02-01 include technical validations (leading to publication of specifications) as well as operational validation involving candidate/representative applications.

The project plans to provide a direct benefit to the SESAR2020 performance targets by the following objectives:

- Making progress with the standardization of the LDACS technology.

\footnotetext{
${ }^{1}$ SESAR2020 refers to sub-projects with the term "solution".

${ }^{2}$ Background information on LDACS can be found in Appendix A.

${ }^{3}$ Note that LDACS was called LDACS1 in SESAR1.
} 
- Development of the LDACS system architecture and finalization of the development of the LDACS air-ground datalink.

- Development and validation of fully functional LDACS prototypes.

The status of each of these objectives is presented in the following sections.

\section{Status of the LDACS Standardization Activities}

Currently, there are no standards and regulations for LDACS available. In general, various standards will need to be developed from different standardization organizations, as prerequisite for the LDACS implementation and deployment. These standards need to cover the frequency aspects (signal in space and interference), performance avionics, etc. It is an objective of PJ.14-02-01 to support the development of LDACS standards.

Considering the currently deployed systems, LDACS standards are expected to be delivered from ICAO, EUROCAE/RTCA, AEEC. In addition, ETSI may also cover LDACS and if in the future there will be a regulation associated with an LDACS deployment in Europe, regulatory standards (i.e., Implementing Rules) may also be required to be developed by the European Commission.

The following paragraphs summarize the documents that each organization may produce and the current status focusing at this stage in ICAO, EUROCAE and AEEC.

The Project Team „Terrestrial Data Link” (PT-T) of the ICAO Communications Panel (CP) expects to develop initial standards by the end of 2018, and initial technical guidance material (Manual) by 2020. The final LDACS SARPS are planned for 2026.

EUROCAE is expected to publish LDACS MOPS to cover the equipment functionality. In addition, a MASPS may also be required to cover the overall system aspects including performance. The involvement of RTCA is desired and a joint activity will be pursued if agreed. EUROCAE together with RTCA have already developed performance and description documents (SPR, OSED) for B2 services and it is expected that they will also develop such documents for the future, more stringent services (B3). The initiation of an LDACS standardization activity in EUROCAE has been discussed and a EUROCAE group to support this is already identified (Working Group 82). However, it has been decided to progress first the standardization work in ICAO before initiating activities in EUROCAE. It is expected that EUROCAE will kick-off LDACS standardization activities at the end of 2018 considering also the progress in ICAO.

AEEC is expected to develop the ARINC avionics standards covering the Form Fit and Function (FFF) aspects of the LDACS avionics box and facilitate the LDACS integration on the aircraft. There have been briefings about LDACS to AEEC and LDACS is identified as a future development activity. Currently it is considered too early to initiate an activity in AEEC. Thus such activity for an ARINC avionics standard will be proposed to commence at a later stage when the ICAO and EUROACE standards will have progressed sufficiently.

\section{Status of the LDACS Development Activities}

LDACS is the technology considered and developed as ground-based part of the Future Communications Infrastructure to support future aeronautical requirements for data communications. Choosing the L-band (960-1164 MHz) for deploying an En-Route terrestrial (long range) communication technology is explained by the lack of suitable frequency bands, as the other two aviation bands are either not available (VHF band is already saturated) or not suitable (propagation/attenuation characteristics of C-band). The L-band which is already extensively used by aviation has been allocated to the Aeronautical Radio Navigation Service for many years. It has also been available for Aeronautical Mobile Route Service since World Radio Conference (WRC) 2007.

LDACS provides high performance by using bandwidth resources more efficiently than existing terrestrial aeronautical datalinks and is well dimensioned to cope with the expected future traffic growth. However as LDACS needs to coexist in the considered band with other legacy aviation systems (such as DME in particular) significant effort is required to demonstrate the compatibility with the 
affected systems and this is an important task that needs to be completed.

The development of LDACS in SESAR2020 is based on previous work performed in recent years: An LDACS specification has been produced [2] and has already been updated; transmitter demonstrators were developed to test the spectrum compatibility of LDACS with existing systems operating in the Lband [3]; and the overall system performance was analyzed by computer simulations, indicating that LDACS can fulfil the identified requirements.

On the basis of these results the development of LDACS is continued in SESAR2020 project PJ.1402-01.

\section{LDACS High-Level Architecture}

The LDACS Access Service Network (LDAN) is defined as a complete set of network functions needed to provide radio access to an LDACS subscriber i.e. an aircraft. The LDAN provides the following mandatory functions: (1) LDACS connectivity between an LDACS airborne station and a LDACS ground station (GS), (2) transfer of AAA messages to LDACS subscriber's home network service provider for authentication, authorization and session accounting for subscriber sessions, (3) network discovery and selection of the LDACS subscriber's preferred network service provider (i.e. IP address allocation) and relay functionality for establishing layer 3 connectivity, and (4) radio resource management. In addition to the above mandatory functions, for a portable and mobile environment, a ground-station controller (GSC) shall support LDAN anchored mobility, a location register, and interface with an Authentication, Authorization, and Accounting (AAA) server.

The LDAN comprises network elements such as one or more Ground Stations, and a Ground-Station Controller. An LDAN may be shared by more than one communication service provider networks. The high-level architecture of the LDACS access network is illustrated in Figure 1.

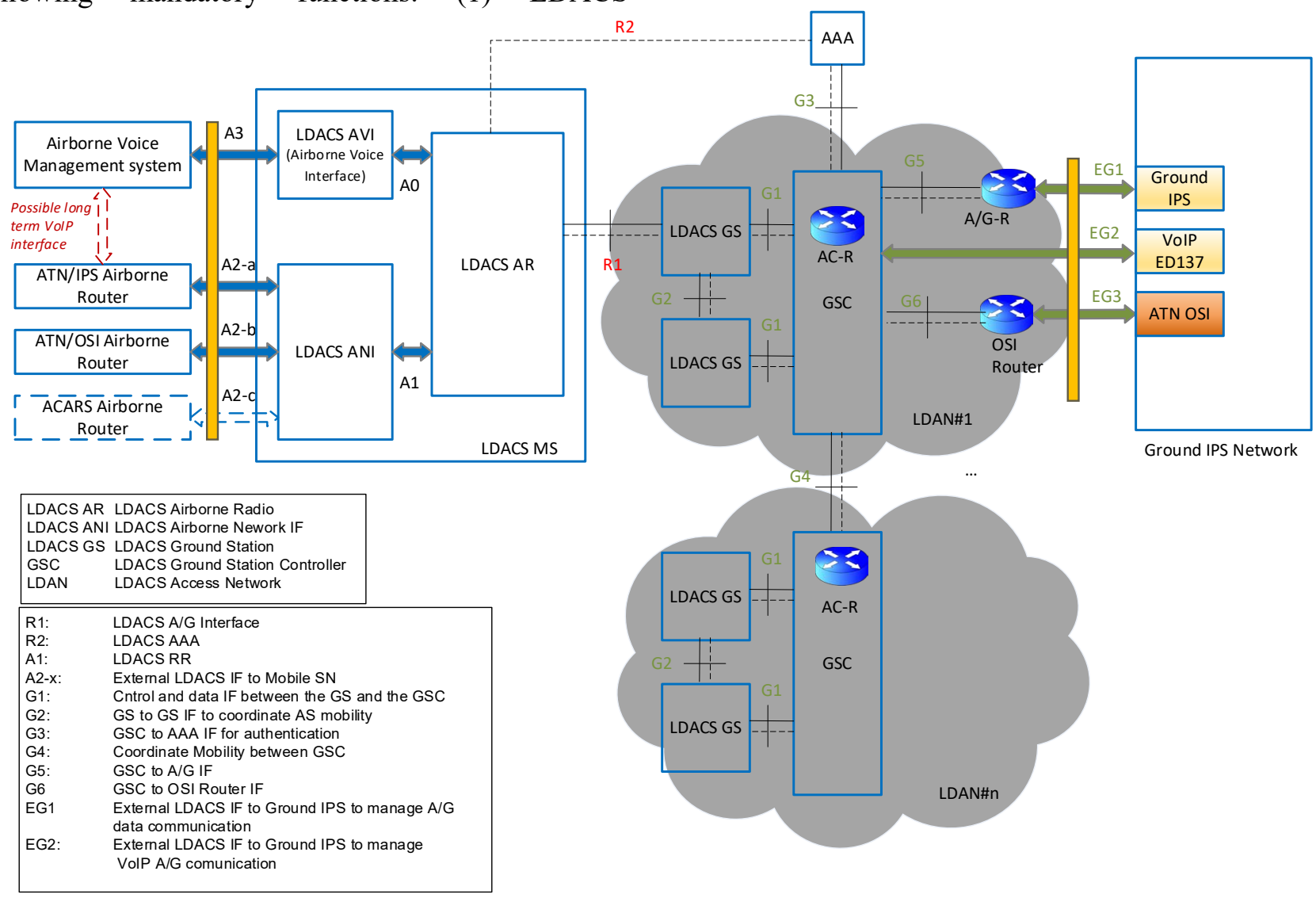

Figure 1. LDACS System Reference Model, Including the LDAN and the LDACS Airborne Subsystem 
The LDACS ground network reference model comprises three entities: Ground-stations, the groundstation controller, the AAA server, and the access and $\mathrm{A} / \mathrm{G}$ router.

Reference point G1 consists of the set of control and user plane protocols for communication between the ground-station and the ground-station controller. The bearer plane consists of intra-LDAN data path between the ground-station and ground-station controller. The control plane includes protocols for data path establishment, modification, and release control in accordance with the aircraft mobility events.

Reference Point G2 consists of the set of control plane protocols originating/terminating in groundstation of an LDAN that coordinate aircraft mobility between ground-stations.

Reference Point G3 is a physical connection and consists of protocols and procedures between AAA server and GSC associated with service authentication, authorization, and accounting management.

Reference Point R1 consists of the protocols and procedures between an aircraft and a ground-station as part of the LDACS air interface (physical layer and medium access layer).

The LDACS airborne reference model comprises three modules: the airborne radio (AR), the airborne network interface (ANI), and the airborne voice interface.

Reference point A1 consists of the set of protocol message that manage the connection between ANI and AR.

Reference point A2-a consists of the set of control and user protocols that manage ATN-IPS airborne router communication. Reference point A2$\mathrm{b}$ consists of the set of control and user protocols that may manage ATN-OSI airborne router communication. Reference point A2-c consists of the set of control and user protocols that may manage ACARS airborne router communication.

Reference point A3 consists of the set of control and user protocols that manages the connection to the airborne voice management system.

\section{LDACS Air-Ground Datalink}

PJ.14-02-01 is currently working on an update of the LDACS specification [2]. Several internal versions were produced and reviewed within the scope of SESAR and were also discussed in ICAO meetings. A first version of the LDACS specification update has been submitted to SESAR in December 2017. Following this version and in preparation of the second version (planned for July 2019), further internal drafts will be produced. The deliverable D3.3.010 "LDACS A/G Specification" of PJ.14-0201 shall then provide the basis for the LDACS manual to be published by ICAO.

\section{LDACS Prototypes}

Project PJ.14-02-01 will develop and verify fully functional LDACS prototypes in order to validate the air interface requirements and to support the validation of ATN IPS infrastructure.

It is planned to execute three different kinds of technical validation exercises. The first type of exercises aims to verify the conformity of the implementation with the existing LDACS specification and should reveal issues in the specification that should be revised when progressing with the LDACS standard. The objective of the second exercise is to interconnect LDACS transmitters and receivers implemented by different Project PJ.14-02-01 members to prove the interoperability of the implemented prototypes and to identify any ambiguity of the LDACS specification. The results of this validation exercise will be used to specify these requirements in the LDACS specification more precise. The third exercise involves two LDACS ground stations and one LDACS airborne station (AS). It aims to demonstrate LDACS' mobility support, including the exchange of LDACS $\mathrm{A} / \mathrm{G}$ mobility management information during the hand-over of an LDACS AS between different GSs. The results from the third validation exercise will be used to verify the proposed mobility solution and assess the impact on LDACS as well as to verify the support of ATS B2 applications using the ATN/IPS ground network.

\section{Planned Technical Validation Exercises}

In order to reach the planned maturity level (TRL4 "technology validated in lab") three different 
kinds of technical validation activities are planned to be performed.

LDACS conformance testing: This activity validates the conformance of the RF front-end and physical layer conformance (transmitter and receiver) with regard to the LDACS specification and the LDACS ranging concept.

LDACS interoperability testing: This activity validates the interoperability of different LDACS prototypes, manufactured by different vendors, over the air interface.

LDACS end-to-end testing: This activity validates the interoperability of LDACS airborne and ground prototypes with ATN/IPS ground network, as required for end-to-end testing. It verifies data link capabilities for air traffic services using ATN/IPS including the verification of airborne and ground IP interfaces. The activity also validates the LDACS mobility support, including the LDACS - A/G mobility management information exchange during LDACS airborne handoff.

\section{Future Work}

While significant progress is expected to be achieved in the context of PJ14-02-01, further work will be required particularly in the area of spectrum compatibility and support to validation and standardization. The SESAR2020 framework foresees that current activities (wave 1) will be completed in 2019 and new activities (wave 2) will be defined to continue the required work (2020 and beyond.),

\section{Conclusion}

The objective of this paper was to provide a status report on the current progress of LDACS research and development within SESAR2020.

SESAR2020 project "PJ.14-02-01 FCI Terrestrial Data Link" is currently developing fully functional LDACS prototypes to validate the air interface requirements and to support the validation of ATN IPS infrastructure. In addition, the project will produce an LDACS architecture and support the standardization of the system. LDACS standardization has started in the context of ICAO.

\section{References}

[1] SESAR, "European ATM Master Plan Executive view." [Online]. Available: https://www.atmmasterplan.eu.

[2] M. Sajatovic, B. Haindl, U. Epple, T. Gräupl, C. Rihacek, M. Schnell, N. Fistas, J.-U. Koch, H.-W. Kim, and E. Le-Ho, "EWA04-1-T2-D1 Updated LDACS1 System Specification," Brussels, Belgium, 2011.

[3] M. Sajatovic, H. Günzel, and S. Müller, "WA04 D22 Test Report for Assessing LDACS1 Transmitter Impact upon DME/TACAN Receivers," Brussels, Belgium, 2014.

[4] ICAO, "Global Air Navigation Plan (GANP)," 2016. [Online]. Available: https://www.icao.int/airnavigation/Documents/GANP -2016-interactive.pdf.

[5] M. Schnell, U. Epple, D. Shutin, and N. Schneckenburger, "LDACS: Future Aeronautical Communications for Air-Traffic Management," Commun. Mag. IEEE, vol. 52, no. 5, pp. 104-110, 2014.

[6] S. Brandes, U. Epple, S. Gligorevic, M. Schnell, B. Haindl, and M. Sajatovic, "Physical Layer Specification of the L-band Digital Aeronautical Communications System (L-DACS1)," in Proceedings of the 2009 Integrated Communications, Navigation and Surveillance Conference, ICNS 2009, 2009.

[7] T. Gräupl and M. Ehammer, "LDACS1 Data Link Layer Evolution for ATN/IPS," in AIAA/IEEE Digital Avionics Systems Conference - Proceedings, 2011.

[8] S. Ayaz, F. Hoffmann, U. Epple, R. German, and F. Dressler, "Performance Evaluation of Network Mobility Handover Over Future Aeronautical Data Link," Comput. Commun., vol. 35, no. 3, pp. 334343, 2012.

[9] N. Franzen, A. Arkhipov, and M. Schnell, "LDACS1 Physical Layer Laboratory Demonstrator," in 2010 Integrated Communications, Navigation, and Surveillance Conference Proceedings, ICNS 2010, 2010.

[10] N. Fistas and M. Schnell, "ICAO Aeronautical Communications Panel (ACP) Fourth Meeting of the 
Working Group of the Whole: LDACS1 Physical Layer Laboratory Demonstrator," 2011.

\section{Appendix A: Background on LDACS}

The L-band Digital Aeronautical Communications System (LDACS) is a broadband air-ground datalink proposed to supplement the VHF communication infrastructure in the L-band [5]. It is designed to provide air-ground data communication with optional support for digital voice. It is a cellular broadband system based on Orthogonal FrequencyDivision Multiplexing (OFDM) technology [6] and supports quality-of-service taking the requirements of aeronautical services into account. It shares many technical features with $3 \mathrm{G}$ and $4 \mathrm{G}$ wireless communications systems.

Computer simulations have assessed the expected performance of LDACS. Multiple independent simulation campaigns were conducted by Gräupl et al. [7], , and Ayaz et al. [8] predicting similar performance figures. Partial prototype implementations in hardware have also been prepared [9] [10].

LDACS will be one of several wireless access networks connecting aircraft to the aeronautical telecommunications network. The LDACS access network contains several ground stations, each of them providing one LDACS radio cell.

The LDACS air interface is a cellular datalink with a star-topology connecting aircraft to groundstations with a full duplex radio link. Each groundstation is the centralized instance controlling all airground communications within its radio cell.

The LDACS air interface protocol stack defines two layers, the physical layer and the data link layer.

The physical layer provides the means to transfer data over the radio channel. The LDACS ground-station supports bi-directional links to multiple aircraft under its control. The forward link direction (FL; ground-to-air) and the reverse link direction (RL; air-to-ground) are separated by frequency division duplex. Forward link and reverse link use a $500 \mathrm{kHz}$ channel each. The ground-station transmits a continuous stream of OFDM symbols on the forward link. In the reverse link different aircraft are separated in time and frequency using a combination of orthogonal frequency-division multiple-access and time-division multiple-access.

Aircraft thus transmit discontinuously on the reverse link with radio bursts sent in precisely defined transmission opportunities allocated by the groundstation.

The data-link layer provides the necessary protocols to facilitate concurrent and reliable data transfer for multiple users. The LDACS data link layer is organized in two sub-layers: The medium access sub-layer and the logical link control sublayer. The medium access sub-layer manages the organization of transmission opportunities in slots of time and frequency. The logical link control sublayer provides reliable and acknowledged point-topoint logical channels between the aircraft and the ground-station using an automatic repeat request protocol.

In depth information on LDACS is available at www.ldacs.com.

\section{Acronyms and Abbreviations}

$\mathrm{A} / \mathrm{C}$

$\mathrm{A} / \mathrm{G}$

AAA

AEEC

AeroMACS

ARINC

AS

ATM

ATN

ATN/IPS

CNS

CP

CS

DLS - IR

EECNS
Aircraft

Air/Ground

Authentication, Authorization, and Accounting

Airlines Electronic Engineering Committee

Aeronautical Mobile Communication System

Aeronautical Radio, Incorporated

Airborne Station

Air Traffic Management

Aeronautical Telecommunications Network

ATN using IPS

Communication, Surveillance

Communications Panel

Community Specification

Datalink Services Implementing Rule

Essential and Efficient Communication Navigation and Surveillance Integrated System 


\begin{tabular}{|c|c|}
\hline ETSI & $\begin{array}{l}\text { European Telecommunications } \\
\text { Standards Institute }\end{array}$ \\
\hline EU & European Union \\
\hline EUROCAE & $\begin{array}{l}\text { European Organization for Civil } \\
\text { Aviation Equipment }\end{array}$ \\
\hline FCI & Future Communications Infrastructure \\
\hline FFF & Form, Fit, and Function \\
\hline GS & Ground-Station \\
\hline GSC & Ground-Station Controller \\
\hline $\mathrm{ICAO}$ & $\begin{array}{l}\text { International } \\
\text { Organization }\end{array}$ \\
\hline IPS & Internet Protocol Suite \\
\hline LDACS & $\begin{array}{l}\text { L-band Digital Aeronautical } \\
\text { Communication System }\end{array}$ \\
\hline LDACS1 & $\begin{array}{l}\text { L-band Digital Aeronautical } \\
\text { Communication System type } 1\end{array}$ \\
\hline LDAN & LDACS Access Service Network \\
\hline MASPS & $\begin{array}{l}\text { Minimum Aviation } \\
\text { Performance Specification }\end{array}$ \\
\hline MOPS & $\begin{array}{l}\text { Minimum Operational Performance } \\
\text { Specification }\end{array}$ \\
\hline OSED & $\begin{array}{l}\text { Operational Services and Environment } \\
\text { Definition }\end{array}$ \\
\hline PT & Project Team \\
\hline RTCA & $\begin{array}{l}\text { Radio Technical Commission for } \\
\text { Aeronautics }\end{array}$ \\
\hline SARPs & Standards and Recommended Practices \\
\hline SESAR & Single European Sky ATM Research \\
\hline SPR & Safety and Performance Requirements \\
\hline VDL2 & VHF Digital Link mode 2 \\
\hline VHF & Very High Frequency \\
\hline VoIP & Voice over IP \\
\hline WRC & World Radio Conference \\
\hline
\end{tabular}

\section{Disclaimer}

Opinions expressed in this work reflect the authors' views only and do not necessarily reflect the views or positions of the organizations they belong to.
This solution, being part of PJ14 "Essential and Efficient Communication Navigation and Surveillance Integrated System - EECNS", has received funding from the SESAR Joint Undertaking under grant agreement No 734168 under European Union's Horizon 2020 research and innovation programme.

\section{Integrated Communications Navigation and Surveillance (ICNS) Conference April 10-12, 2018}

